\title{
Hybrid sensor array for the analysis of Sudan family colorants
}

\author{
Z. Cao ${ }^{1}$, L. Lvova², M. Mastroianni', C. Di Natale ${ }^{3}$, I. Lundström ${ }^{4}$ and R. Paolesse ${ }^{2}$ \\ ${ }^{1}$ Department of Chemistry and Molecular Engineering, East China University of Science and \\ Technology, Shanghai, China \\ ${ }^{2}$ Department of Chemical Science and Technologies, University "Tor Vergata”, Rome, Italy, \\ Ilvova@hotmail.com \\ ${ }^{3}$ Department of Electronic Engineering, University “Tor Vergata”, Rome, Italy \\ ${ }^{4}$ IFM, Linköping University, Sweden
}

\begin{abstract}
:
The application of hybrid sensor array based on pyrrole-substituted porphyrin electropolymers for simultaneous optical and electrochemical analysis of diazo-conjugated dyes of Sudan family is presented. Sudan colorants are widely used in many areas but accurate screening of their consumption is required due to the high toxicity. Thanks to the inherent electrochemical activity of Sudan dyes, as far as the intense coloration, it was possible to find the appropriate conditions of hybrid optical and electrochemical porphyrin electropolymer based sensor array system application for fast and simple monitoring of such analytes.
\end{abstract}

Key words: hybrid sensor array, simultaneous optical and electrochemical analysis, diazo-conjugated Sudan dyes

\section{Introduction}

Having the bright and vivid colors Sudan colorants are widely used in many fields such as household commodities, textile, plastics, etc., in order to improve the luster of commercial products. However the evidences have shown the high toxicity of such colorants that have been classified as carcinogens of third category [1]. For this reason the use of diazo-conjugated colorants as foodstuff or animal feed additives is forbidden. However, the illegal use has been occasionally reported, posing serious risks to public health. The common method of Sudan colorants detection in food matrixes is Liquid Chromatography coupled with UV-Visible spectroscopy (LC-UVvis) or Mass Spectrometry (LC-MS) [2]. Unfortunately this method requires costly equipment and is time consuming. Several researches on electrochemical sensors development for Sudan dyes determination were reported [3-6]. In fact, being Red-ox active species, Sudan dyes can be detected on the appropriate working electrode both via reduction $[3,4]$ or oxidation $[5,6]$ of double $\mathrm{N}-\mathrm{N}$ bond. Another interesting possibility that was shown previously, is chemometric analysis performed on second order spectra data matrix of Sudan colorants obtained from the solvent components gradual change-visible absorption spectra [7]. Nevertheless the possibility of hyphenated optical and electrochemical determination of Sudan colorants in the systems based on the same sensing material layer have not been reported yet.

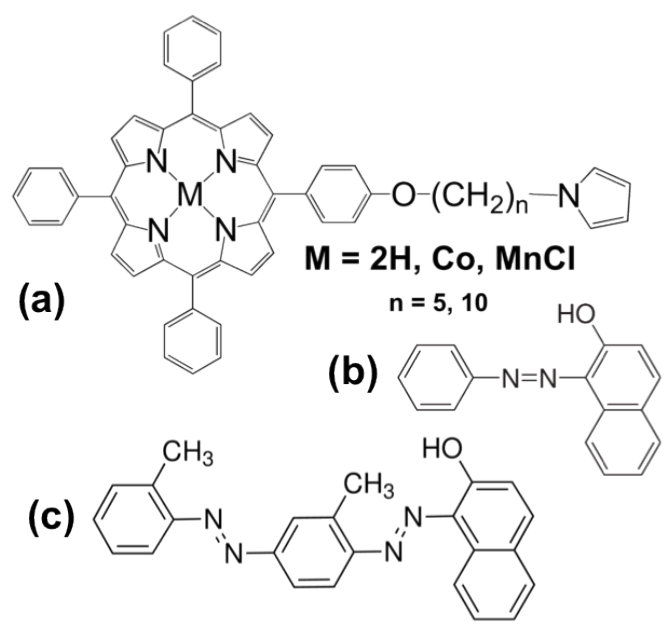

Fig. 1. The chemical structures of (a) mono-pyrrole substituted tetraphenylporphyrins: Mono10 $(\mathrm{M}=2 \mathrm{H}$, $n=10)$, MnMono10 (M=MnCl, $n=10)$, CoMono10 $(M=C o, n=10)$, CoMono5 $(M=C o, n=5) ;$ and analyzed (b) Sudan I and (c) Sudan IV colorants.

Recently we have developed the hyphenated porphyrin-based electropolymers chemical sensors utilizing the same sensing material and 
two different transduction principles: optical and electrochemical [8]. In the present study we report the application of mono-pyrrole substituted tetraphenylporphyrin electropolymers based array for the hyphenated detection of Sudan I and Sudan IV dyes.

\section{Experimental}

The n-alkyl-(1-pyrrole) phenyl-substituted porphyrin monomers and there $\mathrm{Mn}$ (III) and Co (II) complexes were applied for porphyrin electropolymers formation, Fig.1a. The films were obtained by $\mathrm{CV}$ method from $1 \mathrm{mM}$ solutions in $\mathrm{CH}_{2} \mathrm{Cl}_{2}$ solvent containing $0.1 \mathrm{M}$ $\mathrm{TBAClO}_{4}$ supporting electrolyte in standard 3 electrode cell (ITO WE, SCE reference and $\mathrm{Pt}$ counter), and tested by UV-visible spectrometry, AFM and SEM. The response towards Sudan I, IV colorants (Fig.1b,c) was studied by Differential Pulse Voltammetry (DPV, 0 to $-0.8 \mathrm{~V}$ range, $0.025 \mathrm{~V}$ pulse, $0.005 \mathrm{~V}$ step, $\mathrm{N}_{2}$ atmosphere) and Computer Screen Photoassisted Technology (CSPT) in Acetate Buffer Solution (ABS) $\mathrm{pH} 4.7$ in the range $8.6^{*} 10^{-8}$ to $3.8^{*} 10^{-5} \mathrm{M}$. The electroactivity of porphyrin electro-polymers was tested in $0.1 \mathrm{M}$ $\mathrm{Fe}(\mathrm{CN})_{6}^{3-/ 4-}$ probe.

\section{Results and Discussion}

The electroactivity of porphyrin electropolymer films tested in $0.1 \mathrm{M} \mathrm{Fe}(\mathrm{CN})_{6}^{3-/ 4-}$ probe has evidenced that in comparison to the unmodified ITO electrode, the smaller peak separation values were obtained for porphyrin electropolymers (352 mV, $236 \mathrm{mV}, 290 \mathrm{mV}$, and $326 \mathrm{mV}$ for Mono10, MnMono10, CoMono10 and CoMono5 correspondingly versus $463 \mathrm{mV}$ for bare ITO).

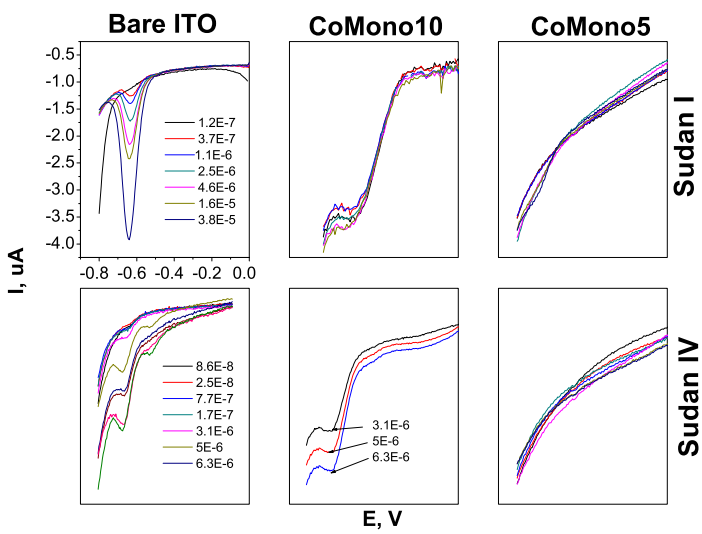

Fig. 2. DPV measurements of Sudan I and Sudan IV dyes on: bare ITO, CoMono10 and CoMono5 films respectively.
These smaller gaps suggest that the electron transfer process is faster on porphyrin electropolymers modified electrodes that permits to consider such materials for red-ox active analytes determination. We then have passed to the optical and electrochemical analysis of Sudan colorants by means of porphyrin electropolymer hyphenated sensor array.
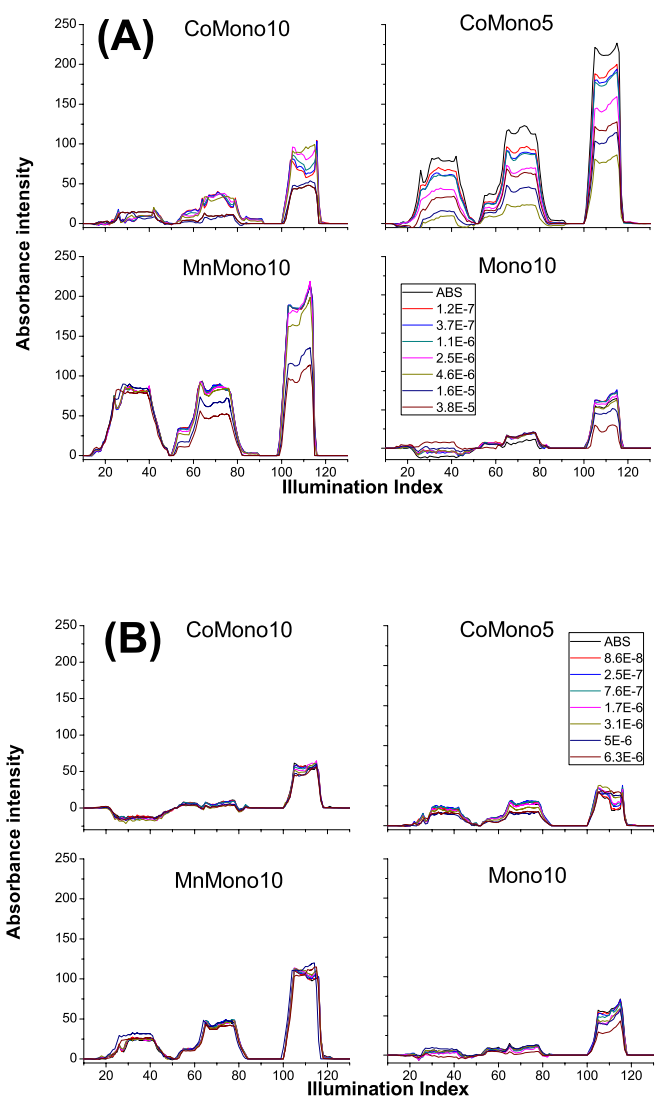

Fig. 3. The CSPT fingerprints of the porphyrin electropolymer films to the different concentration of (A) Sudan I and (B) Sudan IV colorants.

The results of DPV and CSPT tests are shown in Fig. 2 and 3 correspondingly. It has been found that the bare ITO electrode have demonstrated an enhanced DPV response while porphyrin electropolymers have inhibited Sudan electrochemical response in different manner. Thus, no analytically useful response was obtained for Mono10 film due to the central metal acceptor absence; for CoMono10 and CoMono5 the weak reduction waves at $-0.7 \mathrm{~V}$ were obrained, while full electrochemical response quenching due to the $\mathrm{Mn}(\mathrm{III})$ axial coordination by chloride anion was registered for MnMono10 films both in Sudan I and Sudan IV (data not shown). Two reduction peaks at 0.5 and $-0.7 \mathrm{~V}$ corresponding to the rediction 
processes of two double nitrogen-nitrogen bonds could be observed in Sudan IV solutions. Again, the highest DVP response was found for bare ITO, but also CoMono10 film has demonstrated an effective response towards Sudan IV of in $6.3 \times 10^{-6}$ to $3.1 \times 10^{-6} \mathrm{M}$ concentration range, Fig. 2 .

The optical CSPT response of porphyrin films was more effective in comparison to bare ITO and had higher magnitudes in case of Sudan I, Fig. 3. Thus, on the contrary to DPV response CoMono5 and MnMono10 films have demonstrated the higher response absorbing mainly in green and blue regions.

As can be seen from Fig.4, the simultaneous DVP-CSPT measurements has significantly improve the diazo-conjugated colorants classification and has permitted to distinguish the either between Sudan I and IV colorants of different concentrations of the same dye (see Fig.4, bottom).

\section{Conclusions}

In the present study the synthetic requirements and the electrochemical experimental conditions necessary to allow the preparation pyrrole-substituted porphyrin electropolymers were evaluated. Pyrrole-substituted porphyrins were found to be the promising materials for the development of hyphenated optoelectrochemical sensors based on singular sensing layer. The Sudan family dyes were satisfactory discriminated with proposed porphyrin electropolymers hybrid sensor array. CSPT response of porphyrin electropolymers significantly supplements the voltammetric one. The evaluations hybrid porphyrin electropolymers array for determinations of Sudan colorants in food matrices are now in progress in our laboratories.
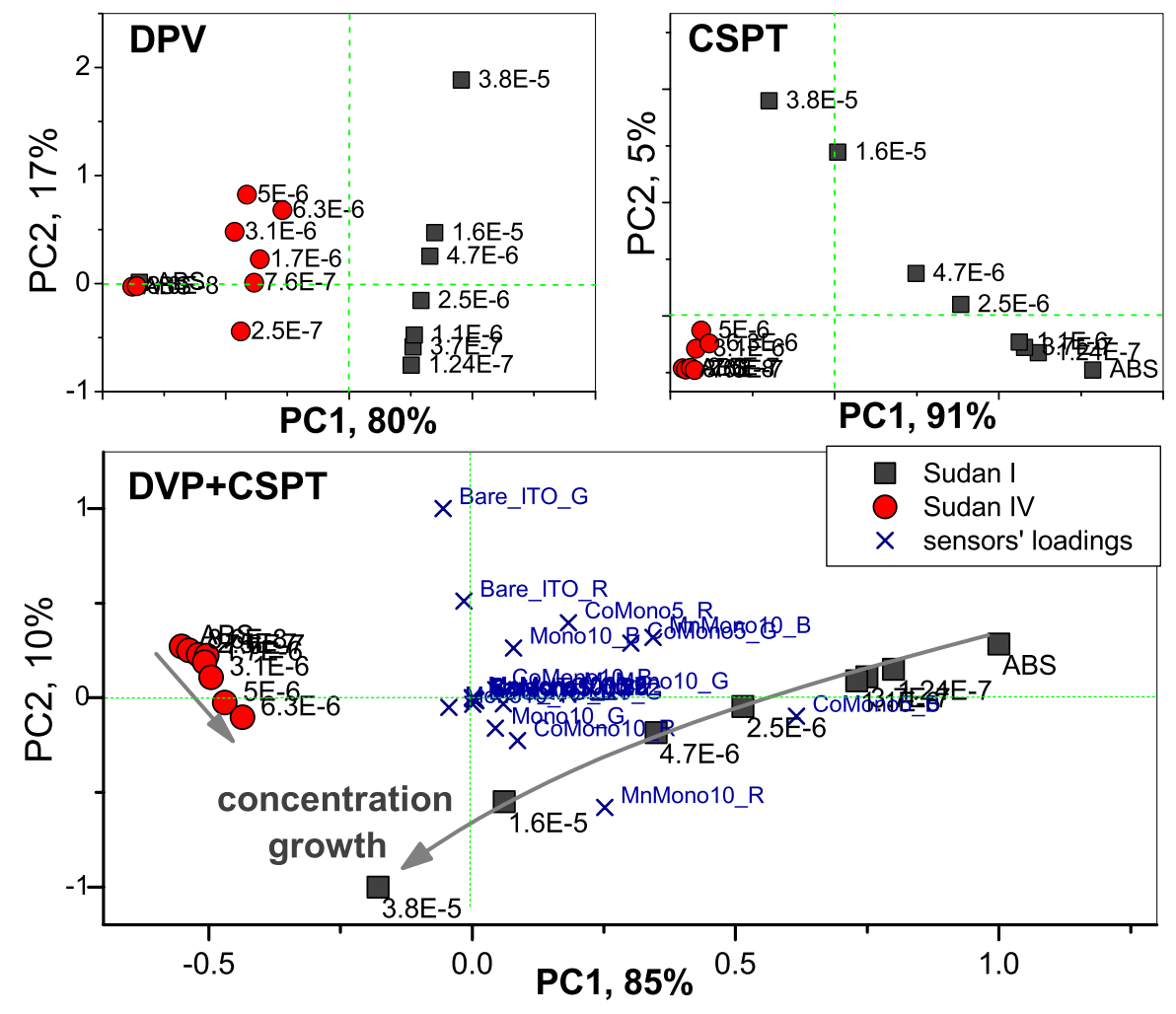

Fig. 4. The PCA classification of Sudan I and IV colorants of different concentrations by CSPT, DPV separately (top) and combined CSPT+DPV analysis (bottom).

\section{Acknowledgements}

L. Lvova would like to acknowledge Prof. B. Floris and Dr. P. Galloni from "Tor Vergata" University, Rome, Italy for provided equipment and $A$. Vecchi for technical support.

\section{References}

[1] L.D. Donna, L. Maiuolo, F. Mazzotti, D.D. Luca, G. Sindona, Anal. Chem. 76, 51045108, (2004) 
[2] R. Rebane, I. Leito, S. Yurchenko, K. Herodes, J. Chromat. A 1217, 2747-2757, (2010)

[3] S. Chen, D. Du, J. Huang, A. Zhang, H. Tu, A. Zhang, Talanta 84, 451-456, (2011)

[4] M.J. Du, X.G. Han, Z.H. Zhou, S.G. Wu, Food Chem. 105, 883-888, (2007)

[5] O. Chailapakul, W. Wonsawat, W. Siangproh, K. Grudpan, Y. Zhao, Z. Zhu, Food Chem. 109, 876-882, (2008)

[6] L. Ming, X. Xi, T. Chen, J. Liu, Sensors 8, $1890-1900,(2008)$

[7] J. Yuan, L. Liao, Y. Lin, C. Deng, B. He, Anal. Chim. Acta 607, 160-167, (2008)

[8] L. Lvova, M. Mastroianni, C. Di Natale, I. Lundström, R. Paolesse, Electroanalysis, 10.1002/elan.201100562. 